\title{
Claude Forest, Patricia Caillé, dirs, Regarder des films en Afrique
}

Lille, Presses Universitaires du Septentrion, 2017, 350 pages

Watch movies in Africa

\section{Hanane ZATOUT}

\section{OpenEdition Journals}

Édition électronique

URL : http://journals.openedition.org/ctd/2136

DOI : $10.4000 /$ ctd. 2136

ISSN : 2491-1437

\section{Éditeur}

Chaire Unesco Pratiques émergentes en technologies et communication pour le développement

\section{Édition imprimée}

ISBN : 2491-1437

\section{Référence électronique}

Hanane ZATOUT, "Claude Forest, Patricia Caillé, dirs, Regarder des films en Afrique », Communication, technologies et développement [En ligne], 7| 2019, mis en ligne le 20 juin 2019, consulté le 24 septembre 2020. URL : http://journals.openedition.org/ctd/2136 ; DOI : https://doi.org/10.4000/ctd. 2136

Ce document a été généré automatiquement le 24 septembre 2020

Communication, technologies et développement 


\section{Claude Forest, Patricia Caillé, dirs, Regarder des films en Afrique}

Lille, Presses Universitaires du Septentrion, 2017, 350 pages

Watch movies in Africa

Hanane ZATOUT

\section{RÉFÉRENCE}

Claude Forest, Patricia Caillé, dirs, Regarder des films en Afrique

Lille, Presses Universitaires du Septentrion, 2017, 350 pages

1 En dépit de la production cinématographique prolifique, les salles de cinéma dans le continent africain font défaut. Ce constat pertinent a nourri la réflexion de plusieurs auteurs réunis dans cet ouvrage sous la direction de Patricia Caillé et Claude Forest. À juste titre, chacun d'entre eux tente de répondre à plusieurs questions : où et comment les films sont vus en Afrique? Quels types de films sont visionnés? Le public visionne-til les films uniquement dans ce dispositif qu'est la salle de cinéma? D'une manière générale, cet ouvrage mène ainsi une réflexion sur les rapports que les spectateurs et spectatrices ont avec les films en Afrique, dans sa diversité, où plutôt en Afrique, comme le souligne le titre. Il tente ainsi d'ajuster la focale sur les films restitués dans leurs contextes de production et de réception.

2 La problématique de cet ouvrage est centrée sur l'accès aux films dans les salles de cinéma par les publics africains. Tandis que les première et deuxième parties, selon une vision à la fois historique et économique, traitent respectivement de l'Histoire de l'Afrique coloniale, puis de la reconstruction des réseaux de distribution et d'exploitation après la décolonisation, la troisième partie est consacrée à des études de pratiques spectatorielles contemporaines dans quatre pays, précisément la Tunisie, le Tchad, le Togo et le Burkina Faso. 
3 De prime à bord, Patricia Caillé souligne la crise du cinéma africain. Si d'habitude, parler du cinéma africain consiste à donner une importance au scénario, aux représentations et aux identités retracées dans chaque œuvre cinématographique, où les cultures africaines et les changements renforcent davantage les frontières politiques entre les pays, mais aussi les barrières psychologiques entre les individus, les spectateurs africains sont relégués au deuxième rang quant à leur appréhension des films. Le propos de cet ouvrage porte donc un regard nouveau sur le cinéma africain sous l'angle des pratiques spectatorielles. L'évocation des spectateurs et spectatrices africains étant plus importante que l'analyse des contenus filmiques, la réflexion de ce volume tend à restituer aux spectateurs leur place.

Ce volume est le fruit de contributions de maints auteurs, chercheurs et professionnels issus de diverses disciplines - histoire, sciences de l'information et de la communication, études filmiques, économie du cinéma, anthropologie, langues et cultures, etc.- réunis lors de projets, séminaires et colloques, notamment la manifestation organisée en mai 2015 à Strasbourg intitulée "Activités et pratiques spectatorielles, publics, cultures du cinéma en Afrique et au Moyen-Orient » qui a clos les activités du projet « MENA Cinémas : cartographie, enjeux, acteurs et publics », ainsi que le colloque «Représentations du cinéma et pratiques spectatorielles en Afrique francophone » organisé en mai 2016 à Marrakech.

\section{Première partie : « Concevoir les publics à partir de l'Histoire »}

5 Dans un article sur « les formes de visibilité de publics africains en Afrique coloniale ", Goerg Odile aborde la question du public et les manières dont il se manifeste en salle et crée une ambiance entre «rire, applaudissement et contestation» (p. 45). Cette entrée renvoie au droit à l'accès aux salles de cinéma, aux modalités de la diffusion en salle de cinéma, comprise comme espace de loisir, et aux formes de sociabilités entre les publics non homogènes.

6 Karine Blanchon s'intéresse, quant à elle, à la programmation des films et aux salles de cinéma à Madagascar. À partir d'un travail sur archives, elle considère que le cinéma national, durant la période coloniale française, était incapable de progresser. En revanche, après l'indépendance, l'industrie nationale a vu le jour grâce à l'émergence de productions vidéo. L'auteure étudie les facteurs politiques, économiques et culturels qui ont conduit à la fermeture et à la transformation des salles vers d'autres activités. La production du film malgache a certes augmenté depuis les années 2000 et ce grâce au développement technologique (caméra numérique...), qui a permis une diffusion sur les écrans à l'étranger, mais connaît malheureusement un ralentissement dans son pays.

7 Nolwenn Mingan, de son côté, s'intéresse à la programmation et à la diffusion du cinéma américain dans le Maghreb durant la période coloniale. Elle pointe les difficultés et les disparités dans la distribution en salles de cinéma dans cette région. L'industrie du cinéma américain et la tarification qu'elle impose sur les places ne crée pas qu'un décalage géographique, mais aussi une séparation des espaces de sociabilité entre les publics. 
Pour sa part, Vincent Bouchard étudie la confrontation de l'image à l'oralité lors de la projection afin de montrer le déplacement médiatique opéré par les pratiques orales sur les films documentaires.

\section{Deuxième partie : «Les pratiques spectatorielles contemporaines »}

9 Patricia Caillé souligne, dans son article, la différence, lors du visionnage des films, entre les spectateurs et spectatrices en Tunisie. De fait, les femmes ne s'expriment pas de la même façon que les hommes sur le plan émotif. Si pour elles, la culture que traduit l'image filmique est source d'inspiration et de fascination, le film reste pour les hommes un moment de divertissement. L'œuvre cinématographique garde son statut en Tunisie et au Maroc où existe une tradition culturelle autour du cinéma.

Claude Forest, qui a fait une synthèse des résultats quantitatifs réalisés en 2015 auprès des spectateurs togolais, souligne le manque de moyens tels que l'électricité, qui rendent l'accès au film difficile. La majorité du public togolais vit en milieu rural, privé de salles de cinéma. La disparition des salles entraîne inéluctablement celle des films. Claude Forest trace ainsi un panorama de l'histoire de l'exploitation du cinéma dans ce pays africain. Il évoque comment les Togolais accèdent aux images soit via un cinéma ambulant soit via la consommation des films dans l'espace privé grâce à l'émergence de la télévision et l'acquisition du lecteur DVD en milieu rural durant ces dernières années.

11 En revanche, le Tchad dépourvu initialement d'infrastructure a connu ces dernières années un développement assez rapide en matière d'énergie électrique grâce aux groupes électrogènes ou panneaux solaires permettant notamment l'accès aux films. Patrick Ndiltah montre que les Tchadiens sont des consommateurs avides d'images. Pour eux, le cinéma est un espace à valeur culturelle et artistique, un vecteur de valeurs religieuses, ou encore un moyen d'éducation.

12 Le dernier article de la deuxième partie, écrit par Justin Ouoro, cerne le nouveau statut du cinéma au Burkina Faso grâce aux entretiens enregistrés auprès des acteurs et des publics. Depuis l'émergence des nouveaux outils audiovisuels, la production cinématographique burkinabée a adopté une nouvelle trajectoire, celle d'exporter des sources de financement local vers une production filmique régulière faite pour plaire à la clientèle. De ce fait, des jeunes acteurs ont redonné vie aux salles de cinéma. L'auteur conclut que les pratiques cinématographiques sont une entreprise commerciale visant principalement, d'une part la satisfaction de la clientèle, d'autre part le gain d'argent. Le cinéma est vu comme lieu de divertissement et non comme un dispositif éducatif.

\section{Troisième partie : «Reconcevoir les publics à partir de l'accès aux films}

Cette dernière partie consiste en un recueil de témoignages d'acteurs de terrain mettant en exergue divers types de production. Michel Serceau, Alessandro Jedlowski et Karine Prévoteau portent leur regard respectivement sur l'offre de films et leur réception dans les salles marocaines de 2006 à 2015, la circulation des vidéos de films 
nigérians en Côte d'Ivoire et les œuvres audiovisuelles africaines, leurs marchés et leurs publics.

Lamia Belkaied-Guiga étudie l'émergence récente de nouvelles technologies favorisant un accès libre à un grand nombre d'œuvres cinématographiques et ce grâce au phénomène de piratage qui a facilité la diffusion de DVD sur le marché parallèle. Dans son travail d'enquête de terrain en Tunisie, elle étudie principalement les dimensions historiques, politiques et socioculturelles qui ont conduit au foisonnement des DVclubs et ont favorisé l'accès libre aux films sans le contrôle de l'État. Dans la deuxième partie de cette enquête, elle cerne les différentes catégories de vendeurs et observe les consommateurs de films piratés pour étudier leurs pratiques cinéphiles. Elle synthétise sa recherche en montrant l'émergence puissante du marché des œuvres cinématographiques piratées, à un point tel que l'État est dans l'obligation de s'y adapter.

Selon Honoré Fouhba, l'apparition des vidéoclubs au nord du Cameroun a constitué un palliatif à la fermeture des salles de cinéma traditionnelles. De ce fait, ces vidéoclubs sont de véritables espaces de médiation qui permettent de rapprocher films et public. Malgré leur interdiction, les vidéoclubs continuent de fonctionner de manière anarchique, contredisant les pratiques de distribution traditionnelles sans contrôle de l'État.

16 Ati Komi souligne quant à lui l'importance des cinémas itinérants dans des pays privés de salle. Ces dispositifs permettent en effet de proposer au public des films différents de ce qu'il à l'habitude de voir (généralement des films importés de l'étranger). À cet effet, il décrit le projet itinérant MobiCiné, qui est destiné à introduire l'éducation par et au cinéma dans les établissements scolaires togolais.

17 Enfin, C. Forest et J. Akouvi Founou font une description détaillée de la programmation filmique au Togo et expliquent notamment comment le cinéma ambulant est organisé. Cette action a été développée dans d'autres pays d'Afrique, entre autres au Bénin, Niger et Mali, afin d'encourager la diffusion du film africain dans des espaces enclavés, de lutter contre le piratage et d'encourager le rassemblement des publics de toute tranche d'âges dans des espaces ruraux au moyen de projections filmiques variées. Sont étudiés non seulement les modes de distribution et de diffusion des films, mais aussi leur réception par les spectateurs.

En somme, outre les efforts qui ont permis d'apporter un éclairage sur l'état actuel des cinémas africains, qui demeuraient jusque-là méconnus, cet ouvrage manifeste également la volonté de créer un réseau pluridisciplinaire réunissant des chercheurs autour des cinémas d'Afrique et du Moyen-Orient. Enfin et surtout, il constituera, à n'en pas douter, une lecture indispensable pour les recherches en Sciences de l'information et de la communication et en Sociologie des publics, qui ne se contentent pas d'étudier les représentations médiatisées par les films, mais cherche à comprendre comment les spectateurs, dans leur diversité, les reçoivent. 


\section{RÉSUMÉS}

Le cinéma dans le continent africain est souvent marginalisé, car la majorité des pays n'ont plus de salle de projection. À ce propos, cet ouvrage rassemble les contributions de chercheurs de différents horizons et champs disciplinaires qui tentent de comprendre les raisons de cette disparition et de présenter les moyens d'y pallier. Surtout, il porte un éclairage sur la façon dont les spectatrices et spectateurs choisissent, regardent, consomment et interprètent les films. Il envisage enfin le rôle des États dans la production et la diffusion des films africains, ainsi que les pratiques spectatorielles dans différents espaces urbains ou ruraux d'Afrique.

Cinema in Africa is often marginalized because the majority of countries no longer have a projection room, in this regard, this book brings together the contributions of researchers from different backgrounds and disciplinary fields where everyone tries to answer questions about the reasons this phenomenon raises the question of how spectators choose, watch, consume and interpret films, the role of the state in the production and distribution of African films, as well as spectatorial practices applied in different urban and rural areas.

\section{INDEX}

Mots-clés : Afrique, cinéma, salle de cinéma, spectateur, réception

Keywords : Africa, cinema, movie theater, spectator, reception

\section{AUTEURS}

\section{HANANE ZATOUT}

Doctorante en Sociologie à l'Université de Reims-Champagne Ardenne (Centre d'études et de recherches sur les emplois et les professionnalisations - CEREP) et à l'Université d'Oran 2 (Laboratoire de Langues, Littérature et Civilisation/Histoire en Afrique - LLCHA). 